\title{
Meiotic Recombination between Dispersed Repeated Genes Is Associated with Heteroduplex Formation
}

\author{
DILIP K. NAG* AND THOMAS D. PETES \\ Department of Biology, University of North Carolina, Chapel Hill, North Carolina 27599-3280
}

Received 13 February 1990/Accepted 18 May 1990

\begin{abstract}
In Saccharomyces cerevisiae, recombination events occurring between allelic genes located on homologous chromosomes are often associated with heteroduplex formation. We found that recombination events between repeated genes on nonhomologous chromosomes (ectopic events) are also associated with the formation of heteroduplexes, indicating that classical and ectopic recombination events involve similar mechanisms.
\end{abstract}

Although studies of meiotic recombination usually concern interactions between DNA sequences located at equivalent positions on homologous chromosomes (interhomolog recombination), recombination between repeated DNA sequences located in nonequivalent chromosomal locations (ectopic recombination) also occurs in yeasts and other eucaryotes (for a review, see references 5 and 11). One specific type of ectopic exchange, recombination between repeated genes on nonhomologous chromosomes (heterochromosomal recombination), has been observed to occur at rates comparable to rates of interhomolog recombination in Saccharomyces cerevisiae $(3,4,7)$. Heterochromosomal crossovers result in reciprocally translocated chromosomes. Below, we describe evidence that many of these exchanges involve a heteroduplex intermediate.

In $S$. cerevisiae, as in many fungi, all four meiotic products can be recovered and analyzed. Although a heterozygous marker usually segregates $2 A: 2 a$ spores $(A$ represents a wild-type allele and $a$ represents the mutant), two types of aberrant (non-2:2) segregation have been observed (for a review, see reference 1). For most alleles, the most common form of aberrant segregation is gene conversion, $3 A: 1 a$ or $1 A: 3 a$ events. These events represent the nonreciprocal transfer of DNA sequences from one allele to the other and are associated with reciprocal exchange about $50 \%$ of the time. In the second type of aberrant segregation, postmeiotic segregation (PMS), one or more spores of the tetrad generate colonies that contain sectors of cells of both genotypes. The most common classes of PMS tetrads are those that contain $2 A$ spore colonies: $1 a$ spore colony: $1 A / a$ sectored colony (called 5:3 segregation) and those that have $1 A: 2 a: 1 A / a$ sectored colony (called 3:5 segregation). These tetrads are likely to reflect the transfer of a single DNA strand from $A$ to $a$ (for 5:3 tetrads) or from $a$ to $A$ (for 3:5 tetrads) (9). PMS events, like conversion events, are associated with reciprocal exchange of flanking markers about half the time (1).

In most models of recombination, heteroduplex formation between interacting homologous sequences is a crucial intermediate in generating a crossover. Since a PMS event represents the segregation of the two alleles from a single haploid meiotic product, such events presumably reflect formation of a heteroduplex between the $A$ and $a$ alleles that contains an unrepaired mismatch. By some models of recombination $(2,9,12)$, gene conversion events are generated by repair of the mismatch in a heteroduplex formed between $A$ and $a$. An alternative possibility is that gene conversion is

\footnotetext{
* Corresponding author.
}

the result of repair of a double-stranded gap in chromosomal DNA (18). Although for most alleles in $S$. cerevisiae gene conversion events are about 10 -fold more common than PMS events (1), we have recently found that strains heterozygous for a mutation in the HIS4 gene (his4-lop) resulting from the insertion of a 26-base-pair palindromic oligonucleotide had twice as many PMS events as conversion events (10). Studies of this mutation, as well as several other types of studies $(1,6,20,21)$, indicate that a substantial proportion of conversion events in $S$. cerevisiae is the result of mismatch repair in a heteroduplex.

The observations described above concern interhomolog recombination. Gene conversion events involving repeats on nonhomologous chromosomes also occur (3), and these conversion events are sometimes associated with reciprocal exchange of flanking markers $(4,7)$. Prior to the study described below, no PMS events involving repeats on nonhomologous chromosomes had been reported. We describe the evidence that such events occur and that these events are associated with crossing over, resulting in reciprocally translocated chromosomes.

Experimental system. In order to look for heteroduplexes formed between repeated genes on nonhomologous chromosomes, we used two related diploid strains, DNY11 and DNY42. The construction of the diploid DNY11 has been described previously (10). The HIS4 genes are located at their normal chromosomal positions in this strain, with one homolog containing the wild-type HIS4 allele and the other containing the his4-lop mutation. This strain has a high rate of non-Mendelian segregation at the HIS4 locus, and about two-thirds of the aberrant segregants represent PMS events (Table 1). The diploid DNY42 is isogenic with DNY11, except for changes introduced by transformation. DNY42 is homozygous for the his4-lop mutant allele at the normal location of the HIS4 gene on chromosome III and contains one copy of the wild-type HIS4 gene inserted within the $A R G 4$ gene on chromosome VIII (Fig. 1). The HIS4 gene on chromosome VIII is oriented in the same direction relative to the centromere as the genes on chromosome III.

If no recombination occurs between the HIS4 genes in DNY42, one expects to get $2 \mathrm{His}+: 2 \mathrm{His}-$ spores. If a heteroduplex is formed as the result of transfer of a single DNA strand of one of the his4-lop genes to one of the wild-type genes, one expects to get $3 \mathrm{His}+: 5 \mathrm{His}-$ segregation (Fig. 1). Transfer of a DNA strand from a wild-type gene to a mutant his 4 gene would be expected to yield either 5 His $+: 3$ His - (if the copy of chromosome III with the heteroduplex cosegregates with the copy of chromosome 
TABLE 1. Aberrant meiotic segregation of HIS4 in strains DNY11 and DNY42 ${ }^{a}$

\begin{tabular}{|c|c|c|c|c|c|c|c|c|}
\hline \multirow{2}{*}{ Strain (type of recombination) } & \multicolumn{5}{|c|}{ No. of tetrads showing indicated segregation ratio } & \multirow{2}{*}{$\begin{array}{l}\text { Total no. } \\
\text { of tetrads }\end{array}$} & \multirow{2}{*}{$\begin{array}{l}\text { Aberrant segregation } \\
\text { (\% of total) }\end{array}$} & \multirow{2}{*}{$\begin{array}{l}\text { PMS (\% of total } \\
\text { aberrant segregants) }\end{array}$} \\
\hline & $5: 3$ & $3: 5$ & $6: 2^{b}$ & $2: 6^{b}$ & $\begin{array}{c}\text { Other } \\
(\text { non-2:2) }\end{array}$ & & & \\
\hline DNY42 (heterochromosomal) & 4 & 16 & $8^{d}$ & $3^{d}$ & $\mathbf{0}$ & 587 & 5 & 65 \\
\hline DNY11 (interhomologous) & 36 & 32 & 23 & 6 & 12 & 312 & 35 & 72 \\
\hline
\end{tabular}

${ }^{a}$ The diploid strains were sporulated at $18^{\circ} \mathrm{C}$ and dissected on plates containing solid rich growth medium (YPD) (15). The spores were allowed to form colonies, and the colonies were replica plated to medium lacking histidine (15). The data concerning DNY11 are taken from reference 10.

${ }^{b}$ The 6:2 and $2: 6$ tetrads are equivalent to the $3: 1$ and $1: 3$ tetrads, respectively.

$c$ Includes tetrads that segregate $4: 0,0: 4,7: 1$, or $1: 7$ or that have more than one PMS event.

${ }^{d}$ Includes both interhomolog and heterochromosomal conversion events.

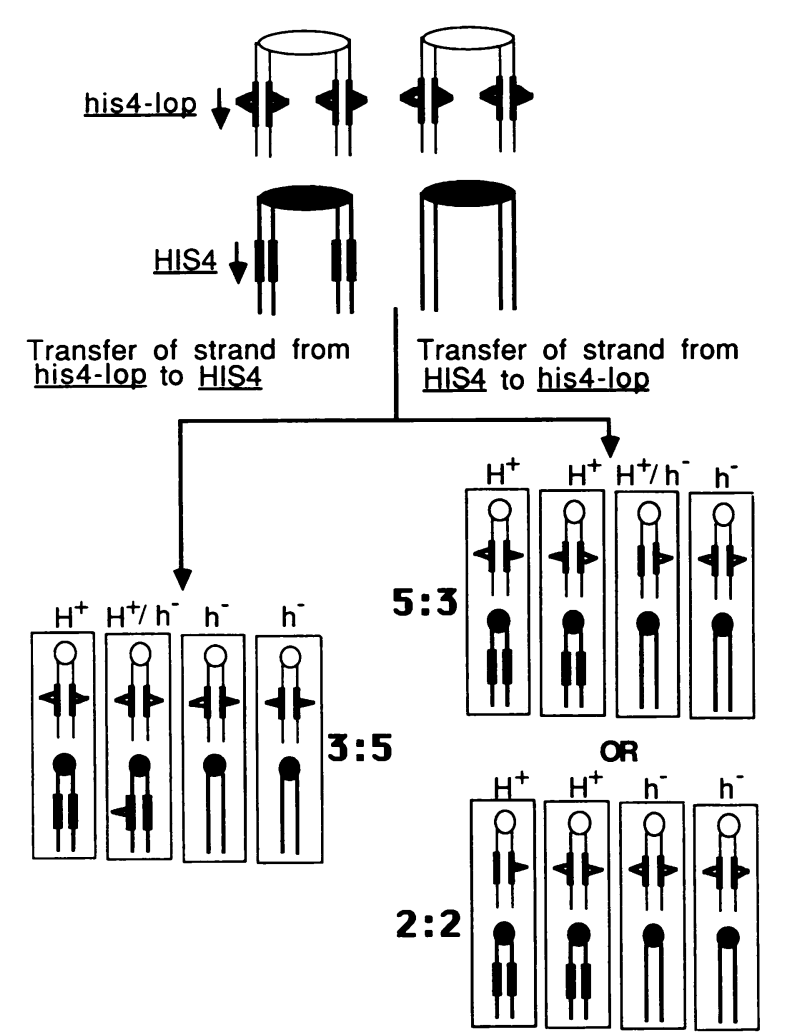

FIG. 1. Expected patterns of PMS for HIS4 genes located on nonhomologous chromosomes in DNY42. The parental haploids used to construct DNY42 were closely related to the strains AS4 ( $\alpha$ trpl-1 arg4-17 tyr7-1 ura3 ade6) and AS13 (a leu2-Bs ura3-52 ade6) used in previous studies $(10,17)$. The haploid strain PD35 (provided by P. Detloff, University of North Carolina, Chapel Hill) was derived from AS4 by a two-step transplacement (22) by using pDN4 (which contains a wild-type URA3 gene and a his4 gene with an insertion of a 26-base-pair palindromic oligonucleotide [called $l o p$ ] in addition to pUC19 DNA sequences). The haploid strain DNY41 was constructed by transforming DNY9 (a derivative of AS13 in which the wild-type HIS4 gene was replaced [14] by a mutant gene that occurred as a result of the insertion of the 26-base-pair palindromic oligonucleotide lop (further details described in reference 10]) with a PstI fragment derived from the plasmid pDN18. The plasmid pDN18 was constructed by inserting a BamHI-Asp 718 fragment (with the sticky ends filled in by using the Klenow fragment of DNA polymerase) containing a wild-type HIS4 gene (derived from plasmid pM45) (10) into the filled-in BglII site in the $A R G 4$ gene of pMLC28ARG4Pst6 (which has a 3.2-kb Pst l fragment containing the $A R G 4$ gene inserted into the PstI site of the M13-derived vector pMLC28; provided by N. Schultes). DNY42 was constructed by mating PD35 to DNY41. This diploid is homozygous for the his4-lop mutation on chromosome III and has a single wild-type copy of
VIII that lacks the HIS4 insertion) or $2 \mathrm{His}+: 2 \mathrm{His}-$ (if the copy of chromosome III with the heteroduplex cosegregates with the copy of chromosome VIII that has the HIS4 insertion). If the two homologs segregate independently, there should be approximately twice as many 3:5 tetrads than 5:3 tetrads. Similarly, heterochromosomal gene conversion events of the 1:3 class would be expected to be observed twice as frequently as such events of the 3:1 class. It should be pointed out that 3:1 and 1:3 tetrads in DNY42 could also result from interhomolog conversion events in which the HIS4 insertion is deleted or duplicated. Thus, the ratio of the two classes of gene conversion events cannot be accurately predicted.

Evidence for heteroduplex formation during ectopic recombination events. About $5 \%$ of the unselected tetrads of DNY42 had an aberrant segregation (Table 1). Approximately two-thirds of the aberrant segregations represent PMS events, and, as expected, there was a significant excess of 3:5 tetrads. The high level of PMS indicates that many heterochromosomal recombination events involve a heteroduplex intermediate. There was an excess of $3: 1$ over $1: 3$ tetrads. A similar bias in favor of $3: 1$ over 1:3 conversions was also observed for interhomolog events involving his4lop (Table 1) in the related heterozygous strain DNY11.

Interhomolog PMS events between the wild-type HIS4 and his4-lop alleles at their normal locations on chromosome III have been previously examined in a diploid strain (DNY11) that is isogenic with DNY42. The frequency of PMS events was about sixfold higher in DNY11 than DNY42, although the fraction of the aberrant segregants that were PMS events was quite similar; correcting for the frequency of ectopic PMS events that cannot be identified (either as the result of segregation patterns that prevent their

HIS4 on chromosome VIII. At the top, both sets of homologs (chromosomes III and VIII are indicated by thin and thick lines, respectively) are shown after meiotic DNA synthesis and before the first meiotic division. The chromosomes are shown as doublestranded DNA molecules, and centromeres are indicated by ovals or circles (open for chromosome III and closed for chromosome VIII). The thicker lines on each chromosome strand indicate HIS4 DNA sequences, with the triangle indicating the mutation in his4-lop. Arrows next to the genes indicate transcriptional orientation. Nonreciprocal transfer of a single DNA strand from one of the his4-lop genes to the HIS4 gene on chromosome VIII (lower left) would result in a 3+:5- segregation pattern. Transfer of a single strand in the opposite direction (lower right) would result in either a $5+: 3-$ or a $2+: 2$ - segregation pattern, depending on which copy of chromosome VIII segregates with the chromosome III that has the heteroduplex. The chromosomes of each spore are enclosed in rectangles. The expected phenotypes of colonies derived from each spore are indicated by $\mathrm{H}^{+}$(His + colony), $\mathrm{h}^{-}$(His- colony), and $\mathrm{H}^{+} / \mathrm{h}^{-}$ (sectored His $+/$ His - colony). 


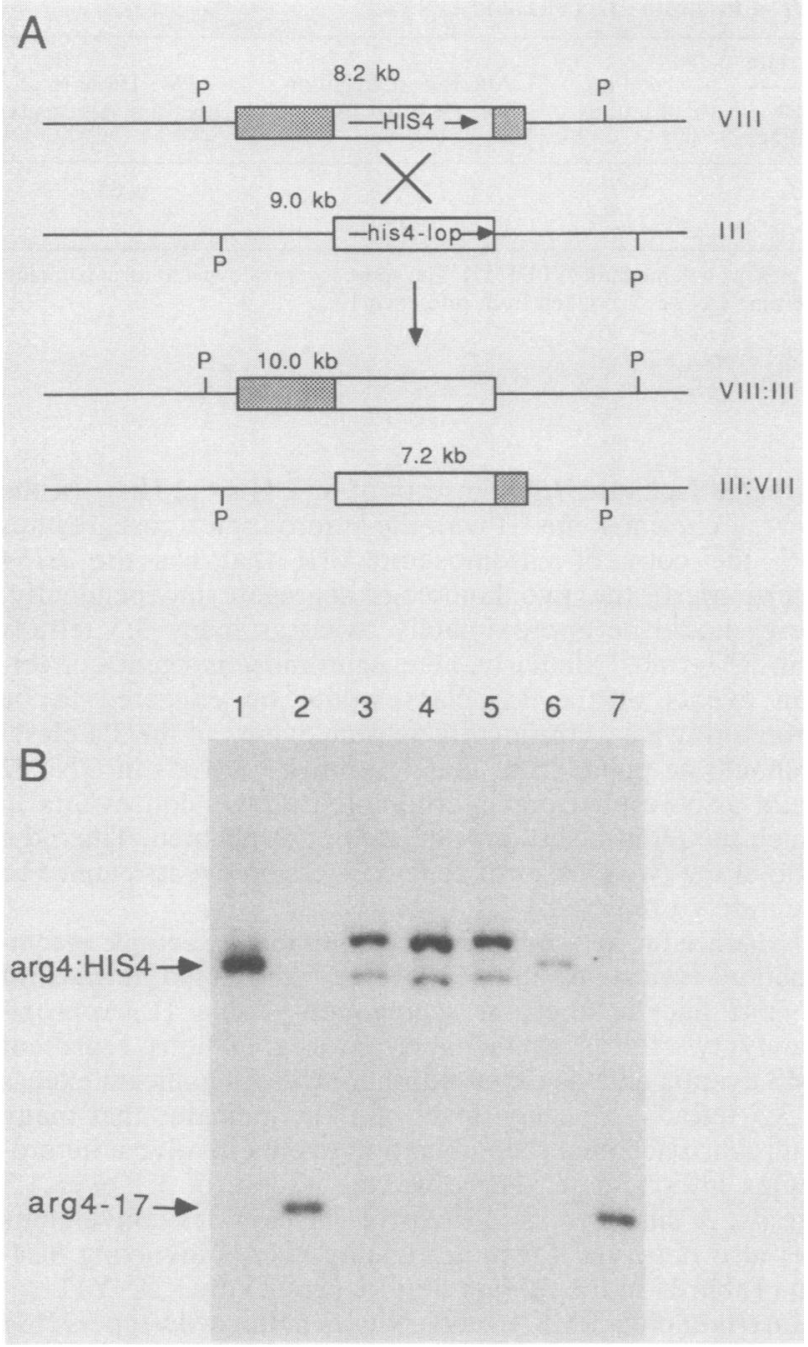

FIG. 2. Physical detection of crossovers between chromosomes III and VIII in DNY42. (A) The positions of PstI (P) sites flanking the HIS4 sequences on the two homologs are indicated. The ARG4 sequences are indicated by the stippled rectangles; the $A R G 4$ gene that does not have an insertion of HIS4 is located on a 3.2-kb PstI fragment that is not depicted in this figure. A reciprocal exchange between the two HIS4 sequences would be expected to produce $P s t \mathrm{I}$ fragments of 7.2 and $10 \mathrm{~kb}$. (B) Southern analysis of DNA isolated from PMS spore colonies derived from DNY42. DNA was isolated (15) from the haploid parents of DNY42 and from cultures derived from spore colonies (taken from YPD plates) exhibiting PMS at the HIS4 locus. The isolated DNA was treated with PstI, and the resulting DNA fragments were examined by Southern analysis, by using the plasmid pMLC28ARG4Pst6 (which has the 3.2-kb PstI fragment containing $A R G 4$ DNA) as a hybridization probe (8). The haploid parental strain DNY41 (lane 1) had the 8.2-kb band expected for the arg4 mutant gene with the integrated HIS4 sequences, whereas the other parental strain (PD35) had the 3.2-kb band expected for the arg4 gene with the point mutation (lane 2). DNA from 3 of 20 sectored colonies (lanes 3 to 5 ) had the $10.0-$ and $7.2-\mathrm{kb}$ bands expected for a reciprocal translocation. DNA from the other colonies yielded either the 8.2 (lane 6)- or the 3.2 (lane 7)-kb fragment.

detection or as the result of associated crossovers that lead to inviable spores), we estimate that interhomolog PMS is about threefold more frequent than heterochromosomal PMS (Table 1). This observation suggests that heterochro- mosomal and interhomolog recombination events are likely to involve quite similar mechanisms.

Evidence that heteroduplex formation during ectopic recombination is associated with crossing over. Interhomolog PMS events are associated with crossing over of flanking markers about half the time (1). Heterochromosomal gene conversion events are also frequently associated with crossing over $(4,7)$. In order to determine whether heterochromosomal PMS events were associated with crossing over of flanking DNA, we examined DNA isolated from spore colonies that contained His $+/ \mathrm{His}-$ sectors. The HIS4 sequences in DNY42 were located on 8.2-kilobase (kb) (chromosome VIII) and 9.0-kb (chromosome III) PstI fragments; $A R G 4$ sequences were either on $8.2-\mathrm{kb}$ (the allele containing the HIS4 insertion) or on 3.2-kb (allele containing the arg4-17 point mutation) Pst I fragments (Fig. 2B, lanes 1 and 2). When ARG4 DNA was used as a hybridization probe to examine DNA from sectored colonies derived from a spore without an associated crossover, single bands corresponding to either the $8.2-$ or $3.2-\mathrm{kb}$ PstI fragments were usually observed (Fig. 2B, lanes 6 and 7). Although only these bands were observed in 17 of 20 PMS events, the two bands expected for an associated reciprocal crossover were detected in three samples (Fig. 2B, lanes 3 through 5).

The frequency of PMS events associated with crossovers $(0.15)$ must be corrected for the fraction of such associated crossovers that result in inviable spores. A heterochromosomal crossover results in two translocated chromosomes, and only spores that receive both translocations would be expected to be viable. If segregation of the recombined products is random, one would expect to recover about one-fourth of associated crossovers (the exact correction factor is described in references 4 and 7). Using the appropriate correction factor, we calculate that about $41 \%$ of the PMS events are associated with crossovers of flanking markers. These results indicate that heteroduplex formation is an intermediate in the production of a heterochromosomal crossover.

If a heteroduplex was formed between arg4-17 and $\arg 4:: H I S 4$ and the resulting large heterology was not repaired, a sectored His $+/ \mathrm{His}$ - spore colony would result. If DNA from such a colony were examined, two PstI fragments of 3.2 and $8.2 \mathrm{~kb}$ would be observed. Since none of the 20 sectored colonies had this pattern, either such heteroduplexes form infrequently or the resulting large heterology is efficiently repaired.

In summary, our results support models of recombination in which heteroduplex formation is an intermediate in the formation of the crossover $(2,9,12)$. The data are also consistent with models in which the lesion that initiates recombination is a double-stranded break that is processed to generate long single-stranded regions $(13,16,19)$.

We thank Malgorzata Dominska for technical assistance, $P$. Detloff for the strain PD35, N. Schultes for the plasmid pMLC28ARG4Pst6, A. Stapleton for the yeast strains AS4 and AS13, and members of the laboratory of Thomas Petes for comments on the manuscript.

This work was supported by Public Health Service grant GM24110 from the National Institute of General Medical Sciences.

\section{LITERATURE CITED}

1. Fogel, S., R. K. Mortimer, and K. Lusnak. 1981. Mechanisms of gene conversion, p. 289-339. In J. N. Strathern, E. W. Jones, and J. R. Broach (ed.), The molecular biology of the yeast Saccharomyces, vol. 1. Cold Spring Harbor Laboratory, Cold Spring Harbor, N.Y. 
2. Holliday, R. 1964. A mechanism for gene conversion in fungi. Genet. Res. 5:282-304.

3. Jinks-Robertson, S., and T. D. Petes. 1985. High frequency meiotic gene conversion between repeated genes on nonhomologous chromosomes in yeast. Proc. Natl. Acad. Sci. USA 82:3350-3354.

4. Jinks-Robertson, S., and T. D. Petes. 1986. Chromosomal translocations generated by high-frequency meiotic recombination between repeated yeast genes. Genetics 114:731-752.

5. Klein, H. L. 1988. Genetic analysis of repeated yeast genes, p. 385-421. In K. B. Low (ed.), The recombination of genetic material. Academic Press, Inc., New York.

6. Kramer, W., B. Kramer, M. S. Williamson, and S. Fogel. 1989. Cloning and nucleotide sequence of DNA mismatch repair gene PMSI from Saccharomyces cerevisiae: homology of PMS1 to procaryotic MutL and HexB. J. Bacteriol. 171:5339-5346.

7. Lichten, M., R. H. Borts, and J. E. Haber. 1987. Meiotic gene conversion and crossing-over between dispersed homologous sequences occurs frequently in Saccharomyces cerevisiae. Genetics 115:233-246.

8. Maniatis, T., E. F. Fritsch, and J. Sambrook. 1982. Molecular cloning: a laboratory manual. Cold Spring Harbor Laboratory, Cold Spring Harbor, N.Y.

9. Meselson, M., and C. Radding. 1975. A general model for genetic recombination. Proc. Natl. Acad. Sci. USA 72:358-361.

10. Nag, D. K., M. A. White, and T. D. Petes. 1989. Palindromic sequences in heteroduplex DNA inhibit mismatch repair in yeast. Nature (London) 340:318-320.

11. Petes, T. D., and C. W. Hill. 1988. Recombination between repeated genes in microorganisms. Annu. Rev. Genet. 22: 147-168.

12. Radding, C. 1982 . Homologous pairing and strand exchange in genetic recombination. Annu. Rev. Genet. 16:405-437.

13. Resnick, M. A. 1976. The repair of double-strand breaks in DNA: a model involving recombination. J. Theor. Biol. 59: 97-106.

14. Rothstein, R. 1983. One-step gene disruption in yeast. Methods Enzymol. 101:202-211.

15. Sherman, F., G. R. Fink, and J. B. Hicks. 1983. Methods in yeast genetics. Cold Spring Harbor Laboratory, Cold Spring Harbor, N.Y.

16. Sun, H., D. Treco, N. P. Schultes, and J. W. Szostak. 1989. Double-strand breaks at an initiation site for meiotic gene conversion. Nature (London) 338:87-90.

17. Symington, L. S., and T. D. Petes. 1988. Expansions and contractions of the genetic map relative to the physical map of yeast chromosome III. Mol. Cell. Biol. 8:595-604.

18. Szostak, J. W., T. L. Orr-Weaver, R. J. Rothstein, and F. Stahl. 1983. The double strand-break repair model for recombination. Cell 33:25-35.

19. Thaler, D. S., and F. W. Stahl. 1988. DNA double-chain breaks in recombination of phage lambda and of yeast. Annu. Rev. Genet. 22:169-197.

20. White, J. H., K. Lusnak, and S. Fogel. 1985. Mismatch-specific post-meiotic segregation frequency in yeast suggests a heteroduplex recombination intermediate. Nature (London) 315:350352.

21. Williamson, M. S., J. C. Game, and S. Fogel. 1985. Meiotic gene conversion mutants in Saccharomyces cerevisiae. I. Isolation and characterization of pmsl-1 and pmsl-2. Genetics 110: 609-646.

22. Winston, F., F. Chumley, and G. R. Fink. 1983. Eviction and transplacement of mutant genes in yeast. Methods Enzymol. 101:211-228. 